\title{
In-situ Raman Spectroscopy in a TEM
}

\author{
F. I. Allen ${ }^{1,2}$, E. Kim ${ }^{3}$, S. Ryu ${ }^{3}$, B. Ozdol ${ }^{1}$, C. P. Grigoropoulos ${ }^{3}$ and A. M. Minor ${ }^{1,2}$ \\ 1. National Center for Electron Microscopy, Lawrence Berkeley National Laboratory, CA, USA \\ 2. Department of Materials Science and Engineering, University of California, Berkeley, CA, USA \\ 3. Department of Mechanical Engineering, University of California, Berkeley, CA, USA
}

Here we report the development of a unique tool to enable in-situ electro-optical characterization and processing of materials at the nanoscale in a dedicated analytical transmission electron microscope (TEM). This work builds upon previous experiments in which an optical fiber was coupled to a TEM using near-field optics to enable the investigation of the microstructural response of materials (imaged using the electron beam) as a result of laser-based nanofabrication processes [1]. Utilizing the near-field approach, optical illumination with a spatial resolution beyond the far-field diffraction limit is achieved. In our new setup, we combine near-field optics with Raman probing to extend the technique to enable photo-excitation and optical characterization, both at high spatial resolution. Furthermore, by installing the assembly onto an analytical TEM incorporating an energy filter, electron energy-loss spectroscopic imaging can be implemented during the in-situ experiments to compliment the photon-based Raman results.

The new assembly is installed on a monochromated $200 \mathrm{kV}$ Zeiss Libra TEM and consists of two components: 1) an in-situ nanomanipulation holder custom-built at LBNL, coupling a lensed optical fiber to the TEM for pulsed near-field illumination, and 2) a Raman probe custom-built by InPhotonics, installed on an adjacent port, with one optical path for an incoming continuous-wave probe laser and a second for the outgoing Raman signals, which are recorded using a Renishaw inVia Raman spectroscope.

Figure 1 presents the first in-situ Raman spectrum acquired using the new setup. This spectrum was obtained upon illumination of a silicon wedge sample (shown in the inset) using a 532nm continuouswave laser beam coupled to the sample chamber via the Raman probe. Using the near-field optical probe, which is coupled to the TEM via the nanomanipulation holder, it is possible to use Raman spectroscopy in combination with TEM imaging to characterize optoelectronic nanostructures such as nanowires with unprecedented spatio-spectral resolution. Various sample geometries can be employed, one example of which is shown in Figure 2. Here, indium nitride nanowires have been grown along the apex of a silicon wedge by laser-assisted chemical vapor deposition [2]. Figure 2a shows a series of localized nanowire growth spots along the wedge and Figure $2 b$ shows several nanowires that have grown from the apex and are thus ideally suited for TEM imaging in combination with near-field optical pulse-probing.

We will present initial results and describe our future plans where we aim to conduct fundamental investigations of laser-induced interactions and processing at the nanoscale, as well as probe electronbeam-induced interactions using the versatility of the Raman spectroscopic technique.

References:

[1] B. Xiang et al., Nano Lett. 12 (2012) p. 2524

[2] D. Hwang et al., Nanotechnology 22 (2011) 385303 
[3] The authors acknowledge funding from a U.S. Department of Energy SBIR grant (DE-FG0207ER84813) awarded to Appliflex, LLC, and thank H. Park \& K. Schriver of Appliflex for their support. The in-situ experiments are performed at the National Center for Electron Microscopy, Lawrence Berkeley National Laboratory, which is supported by the Office of Science, Office of Basic Energy Sciences, Scientific User Facilities Division, of the U.S. Department of Energy under Contract No. DE-AC02-05CH11231.

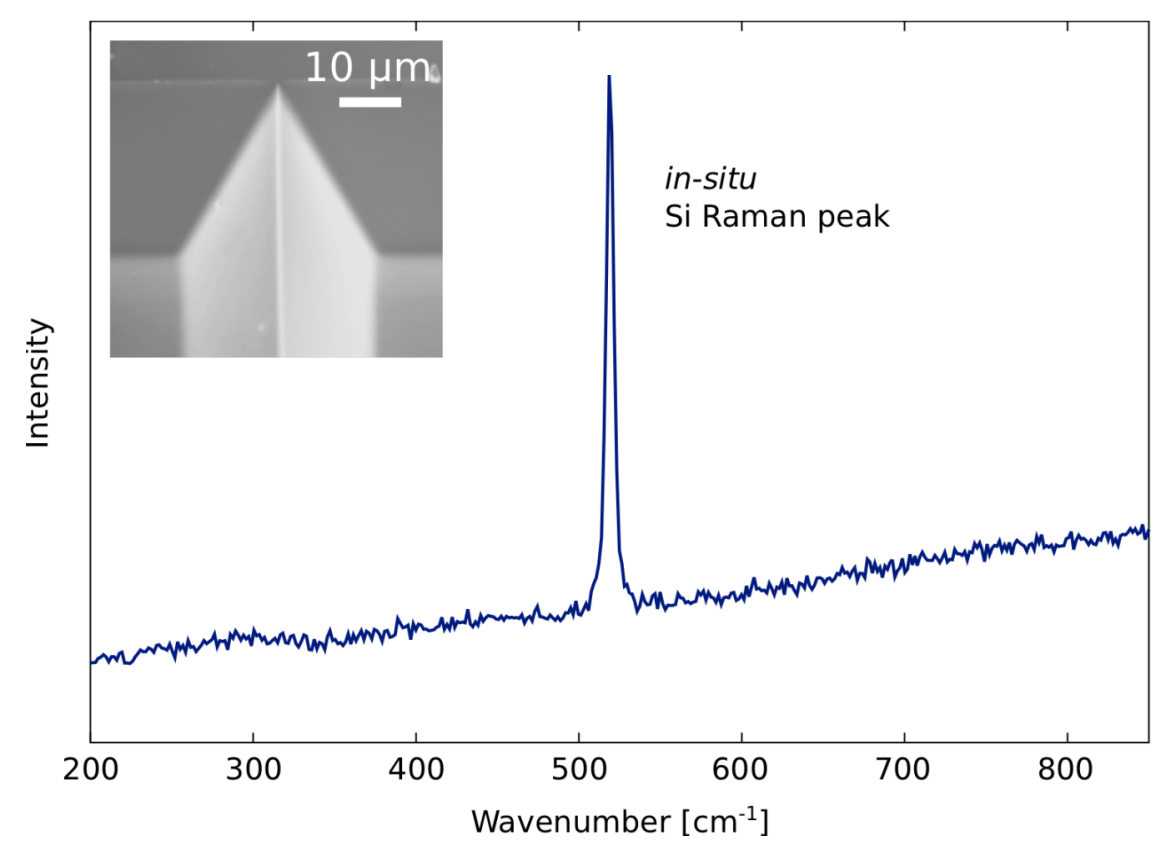

Figure 1 First in-situ TEM Raman spectrum: The characteristic Raman peak for crystalline silicon is recorded for a silicon wedge sample (shown in inset) located inside the TEM.
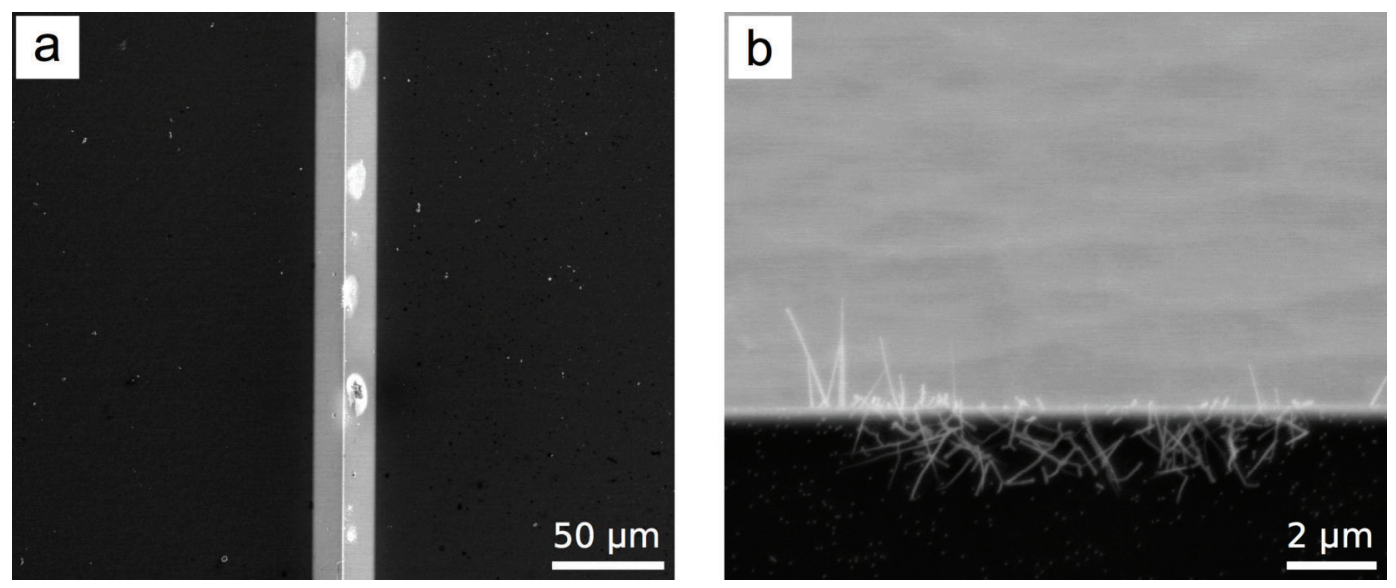

Figure 2a SEM of a silicon wedge viewed from above showing a series of localized laser-assisted indium nitride nanowire growth spots, $\mathbf{b}$ SEM of the same silicon wedge viewed from the side showing indium nitride nanowires at the apex. 